\title{
As obras de edifícios sustentáveis são mais seguras que as construções convencionais? Uma revisão
}

\section{Sustainable building works are safer than conventional constructions? The review}

\author{
${ }^{1}$ Humberto de Campos Costa e Silva 1, ${ }^{2}$ Danilo Ferreira de Souza 2, ${ }^{3}$ Emeli Lalesca Aparecida da Guarda \\ ${ }^{1}$ Especialista em Engenharia de Segurança do Trabalho - Universidade Federal de Mato Grosso \\ (humbertodecampos2001@ hotmail.com) \\ ${ }^{2}$ Doutorando em Energia no Instituto de Energia e Ambiente - Professor Adjunto do Departamento de \\ Engenharia Elétrica da Universidade Federal do Mato Grosso (danilo.ferreira.souza@hotmail.com) \\ ${ }^{3}$ Doutoranda do Programa de Pós-graduação em Arquitetura e Urbanismo - \\ Universidade Federal de Santa Catarina (emeliguarda@gmail.com)
}

RESUMO: Nos últimos anos, verifica-se aumento significativo no número de construções sustentáveis, as quais necessitam de uma série de requisitos para a sua concepção desde a etapa de pré-projeto até a sua demolição. Muitos trabalhadores são envolvidos na etapa de construção, assim, levanta-se o seguinte questionamento: As edificações sustentáveis são mais seguras para os operários durante o processo de construção? Assim, este trabalho tem como objetivo, mapear por meio de revisão bibliométrica, a relação entre certificação de construções sustentáveis e a segurança dos trabalhadores, considerando apenas construções novas. O processo metodológico consiste em revisão da literatura utilizando a metodologia PRISMA. Assim, os artigos analisados demonstraram que, em diversos casos, ocorreu o contrário do esperado: nas obras de edificações certificadas ocorreram maior número de acidentes do trabalho que nas convencionais. Por este motivo, propostas de créditos pilotos para a certificação LEED foram apresentadas, visando a implementação das preocupações com a Segurança e Saúde do Trabalho. Os grupos de pesquisas que publicaram a maior parte dos trabalhos localizados nesta revisão são dos Estados Unidos. Assim, os artigos revisados em sua grande maioria demostram a realidade da sua localidade. Destaca-se que no Brasil, não existem estudos que demonstrem se a etapa de obra das construções sustentáveis são mais seguras do que as convencionais, ou se o número de acidentes do trabalho é maior ou menor nas construções certificadas. Os resultados obtidos revelam que a saúde e segurança do trabalho deve ser parte do sistema de certificação de uma construção sustentável, bem como, os projetos de prevenção segurança e saúde do trabalhador contribuem de forma positiva para diminuição dos riscos. No caso brasileiro, as referências analisadas indicaram que as os procedimentos de certificação aplicados nas obras contribuem positivamente para a melhoria da segurança do trabalhador devido à fiscalização do comprimento das regulamentações estaduais federais e municipais existentes.

Palavras-Chave: Saúde. Segurança. Certificações de Edifícios. Construções Sustentáveis.

\begin{abstract}
In recent years, there has been a significant increase in the number of sustainable constructions, which need a series of requirements for their design, from the pre-project stage to its demolition. Many workers are involved in the construction stage, thus, the following question arises: Are sustainable buildings safer for workers during the construction process? Thus, this work aims to map, through a bibliometric review, the relationship between certification of sustainable constructions and worker safety, considering only new constructions. The methodological process consists of a literature review using the PRISMA methodology. Thus, the articles analyzed showed that, in several cases, the opposite of what was expected occurred: in the works of certified buildings there were a greater number of work accidents than in conventional ones. For this reason, proposals for pilot credits for LEED certification were presented, aimed at implementing the concerns with Occupational Health and Safety. The research groups that published most of the works found in this review are from the United States. Thus, the articles reviewed mostly demonstrate the reality of your location. It is noteworthy that in Brazil, there are no studies showing whether the construction stage of sustainable constructions is safer than conventional constructions, or whether the number of work accidents is higher or lower in certified constructions. The results obtained show that health and safety at work must be part of the certification system for a sustainable construction, as well as the prevention projects, safety and health of the worker, contribute in a positive way to reduce risks. In the Brazilian case, the analyzed references indicated that the certification procedures applied in the works contribute positively to the improvement of worker safety due to the inspection of compliance with the existing federal and municipal state regulations.
\end{abstract}

Keywords: Health. Safety. Building Certifications. Sustainable Buildings. 
Silva, H. C. C.; Souza, D. F.; Guarda, E. L. A.; As obras de edifícios sustentáveis são mais seguras que as construções convencionais? Uma revisão. ES - Engineering and Science, 2021, 10:3.

\section{INTRODUÇÃO}

A construção civil é um dos setores que mais consome recursos naturais e, consequentemente impacta negativamente com o meio ambiente em toda sua cadeia produtiva (FARIA e SCHMID, 2015). Neste contexto, utiliza cerca de 60\% das matérias primas disponíveis no planeta (CAMPOS, 2012), 40\% do total de energia produzida (INGRAO et al., 2016) e em 2010 emitiu 8,8 GtCO2eq. Segundo a United States Environmental Protection Agency (EPA) (2014), o setor da construção é responsável por consumir $41 \%$ de toda energia produzida e $13 \%$ de água tratada, além de emitir $37 \%$ dos gases de efeito estufa.

Desde o início da década de 90, tem se discutido com mais frequência sobre os mecanismos para diminuir o impacto causado pela humanidade no meio ambiente. Os resultados destes debates foram as criações de estratégias, padrões, regras e procedimentos para a utilização de recursos de forma mais eficiente na construção civil. Dessa evolução surgiu o termo edifícios sustentáveis. A EPA (2014) conceitua edifícios sustentáveis como a prática de criar estruturas, usando processos ambientalmente responsáveis e recursos com eficiência ao longo do ciclo de vida da edificação, desde sua localização, projeto, operação, manutenção, reforma e demolição. Os edifícios sustentáveis também são conhecidos como edifícios verdes ou eficientes ou de alta performance. A partir dessas estratégias estabelecidas começaram a surgir as certificações de construções consideradas sustentáveis em diversos países no mundo, tais como, na Inglaterra, o BREEAM (Building Research Establishment Environmental Assessment Method) em 1990, nos Estados Unidos, o LEED (Leadership in Energy and Environmental Design) em 1993, na Alemanha, o DGNB (German Sustainable Building Council) em 2007, no Brasil, o Processo AQUA-HQE Construção Sustentável em 2007 e o GBC Brasil Casa e Condomínio, em 2013.

De acordo com o United State Green Building Council (USGBC) (2021), os critérios avaliados pela certificação LEED considerando todo o ciclo de vida dos edifícios, são: processo integrado, localização e transporte, terreno sustentável, eficiência hídrica, energia e atmosfera, materiais e recursos, qualidade do ambiente interno, inovação e prioridade regional. O BRE (2018) (Building Research Establishment) utiliza na certificação BREEAM considerando todo o ciclo de vida dos edifícios, os critérios de: gerenciamento, saúde e bem-estar, energia, transporte, água, materiais, resíduos, uso da terra e ecologia, poluição e inovação. Para a DGNB (2018), um sistema de avaliação deve prezar pela qualidade ambiental, econômica, sociocultural e funcional, técnica, do processo e da localização. Já para a Fundação Vanzolini (2016), detentora do Processo AQUA-HQE, a qual aplica o conceito de relação do edifício com seu entorno, a escolha integrada de produtos, sistemas e processos construtivos, canteiro de obra de baixo impacto ambiental, gestão da energia, água e resíduos, manutenção, conforto higrotérmico, acústico, visual e olfativo e qualidade sanitária dos ambientes, do ar e da água, são fundamentais para a qualidade da edificação. Por fim, segundo GBC (2017), são julgados os critérios de sustentabilidade pela implantação, uso eficiente da água, energia e atmosfera, materiais e recursos, qualidade ambiental interna, requisitos sociais, inovação e projeto. Certificações GBC Brasil Casa e Condomínio, utilizam-se de créditos (ações sugeridas) para pontuaram e assim alcançar o nível desejado (Certificado, Prata, Ouro e Platina). Destaca-se que o Brasil é o quarto país que mais possui edifícios certificados em nível mundial (CBG, 2018).

Segundo NIOSH (2010), a certificação LEED não aborda ou menciona em nenhum dos seus créditos ou pré-requisitos quanto à segurança dos trabalhadores na etapa de execução da obra. Seriam necessárias ações para melhorar a sinergias e benefícios entre a construção sustentável e a segurança ocupacional dos trabalhadores, tais como: trocas de informações, iniciativas com crédito piloto, sistema de classificação de saúde e prevenção e integração de segurança e saúde ocupacional nas análises de ciclo de vida da edificação. 
O diálogo entre o USGBC e NIOSH, resultou na análise um crédito piloto "LEED Pilot Prevention through Design (PtD)". De acordo com USGBC (2015), esse crédito piloto pode identificar medidas de proteção adicionais para melhorar a segurança e a saúde dos trabalhadores da construção. Já no Brasil, de acordo com o Anuário Estatístico de Acidentes do Trabalho (AEAT) (2018), foram registrados 9.291 casos de acidentes do trabalho na indústria da construção civil, sendo 7.794 com registradas na Comunicação de Acidente de Trabalho (CAT) e 1.497 sem registro de CAT.

Neste contexto, são realizadas poucas pesquisas que relacionam a segurança dos trabalhadores em obras de edifícios em processo de certificação. Por este motivo, este trabalho visa responder os seguintes questionamentos:

- Os edifícios em processos de certificação impactam de forma positiva na segurança e saúde dos trabalhadores?

- A segurança e a saúde dos trabalhadores deveriam ser parâmetros a ser considerados nas certificações?

Assim, este trabalho tem como objetivo, mapear por meio de revisão bibliométrica a relação entre certificação de construções sustentáveis com a segurança dos trabalhadores, considerando construções novas.

\section{MATERIAIS E MÉTODOS}

Elaborou-se uma revisão de literatura cujo tema abordam segurança do trabalho em edifícios que visam certificações em construções sustentáveis. O método de pesquisa adotado baseia-se no Preferred Reporting Items for Systematic Reviews and Meta-Analyses (PRISMA). Conforme Moher et al. (2009) o método estruturado de organização de informações que consiste na aplicação de fluxograma de quatro etapas (identificação, seleção, elegibilidade e inclusão), o qual resulta em maior velocidade de leitura, e análises das informações. Esta metodologia é amplamente aplicada desde 2009 por diversos pesquisadores e sua eficácia comprovada uma vez que possui mais de 73.000 citações localizadas pela plataforma Google Scholar, e mais de 24.000 citações localizadas pela plataforma PLOS Medicine. Assim, aplicou-se processo metodológico de acordo com a Figura 1.

Figura 1 - Fluxograma do processo metodológico da revisão
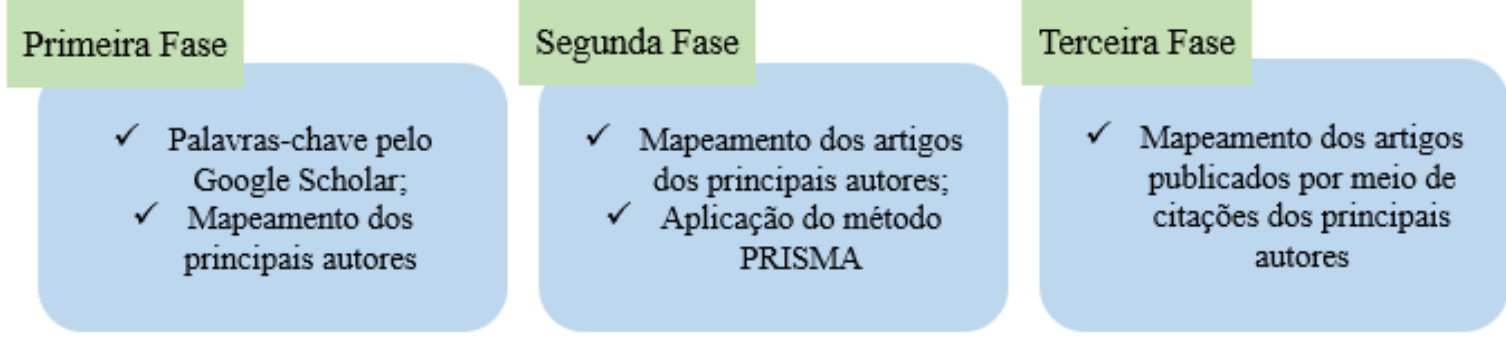

Na primeira fase, foi utilizado o mecanismo de busca Google Scholar, com finalidade de mapear apenas os principais autores das publicações nesse segmento. As pesquisas se deram com palavras-chave, soltas e/ou concatenadas: acidente, trabalho, acidente trabalho, obra certificada, construção sustentável, safety, sustainable, construction, healthy, risk, green construction, development e green building.

Foi escolhido o Google Scholar como máquina de busca, pela simplicidade que a plataforma apresenta, e segundo Martín (2018) a plataforma é potencialmente a mais abrangente da literatura científica e acadêmica em comparação com as duas principais bases de dados multidisciplinares existentes com políticas de inclusão baseadas em periódicos seletivos, como, por exemplo, a Web of Science (WoS) e Scopus. 
A primeira fase retornou 29.000 (vinte e nove mil) resultados utilizando as palavraschave acima. Foram analisados os primeiros 300 títulos que estavam relacionados a pergunta central da pesquisa, com a finalidade de mapear os autores que mais possuem citações. $\mathrm{O}$ mapeamento dos nomes partiu da análise das citações e das referências bibliográficas. Desta forma, primeiramente realizou-se as buscas por autores com pesquisa intrinsecamente ligadas ao tema e na segunda fase, iniciou-se o mapeamento por artigos dos seis autores e citações dos principais artigos encontrados na fase anterior, e não mais pelas palavras chaves.

Iniciou-se a etapa de identificação do método PRISMA. As pesquisas encontraram 560 publicações onde foram selecionados 35 artigos para a leitura completa. Dos 35 artigos selecionados, apenas 15 foram incluídos na análise.

A Figura 2, demonstram os resultados das etapas 2 e 3 agrupadas.

Figura 2 - Etapas da metodologia PRISMA aplicada neste estudo.

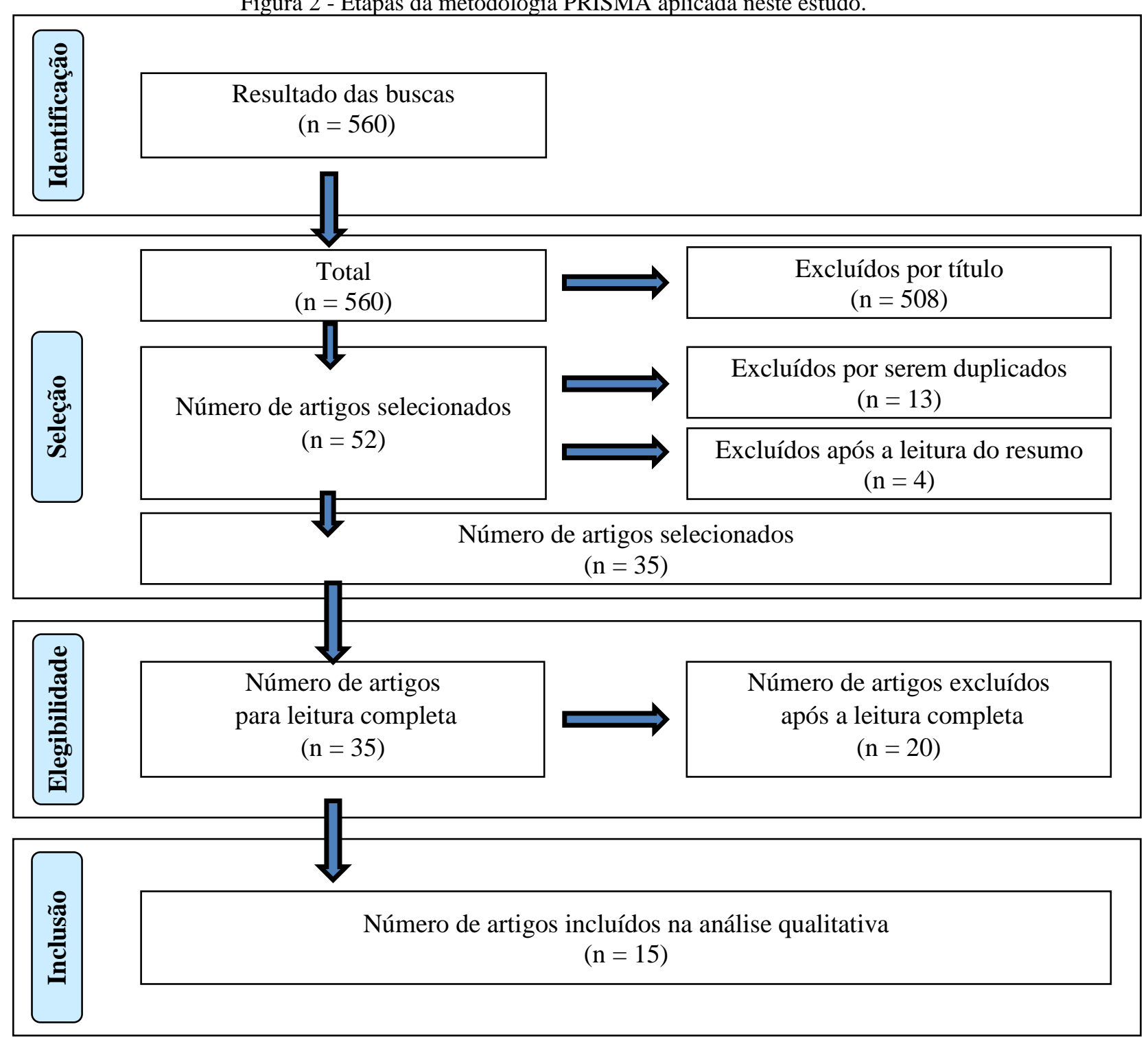

Para a referida pesquisa não foi feito recorte temporal. Entretanto, as obras certificações nas edificações a partir de meados dos anos 90 . Em seguida são apresentados os resultados das análises qualitativa e quantitativa dos 15 documentos. 
Na etapa de Identificação dos trabalhos que pudessem compor este estudo de revisão foram aplicadas as palavras chaves utilizando da máquina de busca Google Scholar, conforme os critérios mencionados.

Na etapa de Seleção foi realizada a leitura detalhada dos títulos e resumos, excluindose os artigos duplicados e os que não abordassem a relação entre edificação certificadas e a segurança do trabalho, portanto uma análise qualitativa.

$\mathrm{Na}$ etapa de Elegibilidade foi realizada a leitura completa dos artigos, e excluindo os trabalhos que não apresentassem em seu conteúdo, contribuições com a discussões edificação certificadas e a segurança do trabalho, ou indicadores que pudessem relacionar os temas.

$\mathrm{Na}$ etapa de Inclusão dos trabalhos, foram selecionados os artigos nos quais identificaram indicadores específicos da área da saúde e segurança ocupacional relacionados a edificações certificadas partindo, portanto, para a análise qualitativa detalhada dos trabalhos selecionados.

\section{RESULTADOS/ DISCUSSÕES}

\subsection{Análise Bibliométrica}

O mapeamento dos nomes partiu da análise das citações e das referências bibliográficas. Assim foi possível encontrar incialmente seis autores citados na Tabela 1.

Tabela 1 - Nome dos principais autores e número de artigos publicados utilizados nas buscas

\begin{tabular}{lc}
\hline Autor & Artigos \\
\hline GAMBATESE, J. A. & 8 \\
BEHM, M. & 5 \\
RAJENDRAN, S. & 4 \\
HALLOWELL, M. & 4 \\
KARAKHAN, A. A. & 2 \\
FORTUNATO, B. R. & 2 \\
\hline
\end{tabular}

Na segunda fase, iniciou-se o mapeamento por artigos dos seis autores encontrados na fase anterior, e não mais pelas palavras chaves.

A Tabela 2 demonstra os artigos, periódicos e fatores de impacto (FI). O fator de impacto é método utilizado que avalia a qualidade da revista científica através das citações recebidas que estejam indexadas na coleção Web of Science. Nota-se que maioria das publicações analisadas foram coletadas de periódicos que possuem alto FI, ou seja, possui alta abrangência entre o meio científico.

Conforme Tabela 2, verificou-se a quantidade de publicações anuais sobre o tema. Essa análise revelou que 73,33\% das publicações ocorreram até o ano de 2013. Observa-se assim, que o interesse em estudar o impacto da Segurança e Saúde do Trabalhador (SST) em obras sustentáveis acompanha o crescimento do número de solicitações de registros no USGBC. Conforme USGBC (2016), entre os anos de 2000 e 2006 eram registrados em média 60 projetos/mês em nível mundial. Já nos anos de 2007 e 2008, os números de registros subiram para aproximadamente 700 projetos/mês. 
Silva, H. C. C.; Souza, D. F.; Guarda, E. L. A.; As obras de edifícios sustentáveis são mais seguras que as construções convencionais? Uma revisão. ES - Engineering and Science, 2021, 10:3.

Tabela 2 - Título/Periódico/Ano/Citações (Set 2021) / Fator de Impacto - dos 15 artigos selecionados

\begin{tabular}{|c|c|c|c|c|c|}
\hline & Documento & Periódicos & Ano & Citações & FI \\
\hline 1 & $\begin{array}{l}\text { Development and Initial Validation of Sustainable } \\
\text { Construction Safety and Health Rating System }\end{array}$ & $\begin{array}{l}\text { Journal of Construction } \\
\text { Engineering and Management }\end{array}$ & 2009 & 163 & 3.38 \\
\hline 2 & $\begin{array}{l}\text { Impact of Green Building Design and Construction } \\
\text { on worker safety and health }\end{array}$ & $\begin{array}{l}\text { Journal of Construction } \\
\text { Engineering and Management }\end{array}$ & 2009 & 130 & 3.38 \\
\hline 3 & $\begin{array}{l}\text { Identification of Safety Risks for High-Performance } \\
\text { Sustainable Construction Projects }\end{array}$ & $\begin{array}{l}\text { Journal of Construction } \\
\text { Engineering and Management }\end{array}$ & 2012 & 109 & 3.38 \\
\hline 4 & $\begin{array}{l}\text { Prevention through design and construction safety } \\
\text { management strategies for high performance } \\
\text { sustainable building construction }\end{array}$ & $\begin{array}{l}\text { Construction Management and } \\
\text { Economics }\end{array}$ & 2012 & 72 & 2.45 \\
\hline 5 & $\begin{array}{l}\text { Safety Risk Quantification for High Performance } \\
\text { Sustainable Building Construction }\end{array}$ & $\begin{array}{l}\text { Journal of Construction } \\
\text { Engineering and Management }\end{array}$ & 2012 & 57 & 3.38 \\
\hline 6 & $\begin{array}{l}\text { Integrating worker health and safety into sustainable } \\
\text { design and construction: designer and constructor } \\
\text { perspectives }\end{array}$ & $\begin{array}{l}\text { Journal of Construction } \\
\text { Engineering and Management }\end{array}$ & 2017 & 42 & 3.38 \\
\hline 7 & $\begin{array}{l}\text { Green design \& construction understanding the } \\
\text { effects on construction worker safety and health }\end{array}$ & Professional Safety & 2007 & 38 & $-*$ \\
\hline 8 & $\begin{array}{l}\text { Identification, Quantification, and Classification of } \\
\text { Potential Safety Risk for Sustainable Construction in } \\
\text { the United States }\end{array}$ & $\begin{array}{l}\text { Journal of Construction } \\
\text { Engineering and Management }\end{array}$ & 2017 & 32 & 3.38 \\
\hline 9 & $\begin{array}{l}\text { Prevention through Design and green buildings-A } \\
\text { US perspective on collaboration }\end{array}$ & (CIB) W99 Conference & 2009 & 13 & $-*$ \\
\hline 10 & $\begin{array}{l}\text { Sustainable buildings: Applying prevention through } \\
\text { design }\end{array}$ & Professional Safety & 2013 & 9 & $-*$ \\
\hline 11 & $\begin{array}{l}\text { LEED Credits: How They Affect Construction } \\
\text { Worker Safety }\end{array}$ & Professional Safety & 2012 & 7 & $-*$ \\
\hline 12 & Building Toward Sustainable Safety and Healthy & $\begin{array}{l}\text { American Society of Safety } \\
\text { Engineers }\end{array}$ & 2006 & 4 & $-*$ \\
\hline 13 & $\begin{array}{l}\text { Adopting green construction practices: health and } \\
\text { safety implications }\end{array}$ & $\begin{array}{l}\text { Journal of Engineering, Design } \\
\text { and Technology }\end{array}$ & 2019 & 9 & 0.324 \\
\hline 14 & $\begin{array}{l}\text { Sustainability Measures and Potential Impacts on } \\
\text { Occupational Safety and Health: Brazilian } \\
\text { Constructions }\end{array}$ & $\begin{array}{l}\text { Electronic Journal of } \\
\text { Geotechnical Engineering }\end{array}$ & 2014 & 2 & 0.11 \\
\hline 15 & Green and Healthy Jobs & $\begin{array}{l}\text { Labor Occupational Health } \\
\text { Program at the University of } \\
\text { California at Berkeley }\end{array}$ & 2010 & 1 & $-*$ \\
\hline
\end{tabular}

*não apresenta

A Figura 3 mostra uma redução de publicações em sua tendência (linha pontilhada). Somente do ano de 2009, houve 279 citações correspondendo 45,51\% do total. Esse interesse provavelmente originou-se do crescimento exponencial nos números de solicitações de registros no USGBC, pelo fato que as pesquisas estão sendo difundidas no EUA, onde outras certificações não são tão difundidas, como o BREAM por exemplo. 
Silva, H. C. C.; Souza, D. F.; Guarda, E. L. A.; As obras de edifícios sustentáveis são mais seguras que as construções convencionais? Uma revisão. ES - Engineering and Science, 2021, 10:3.

Figura 3 - Número de publicações anuais e a tendência

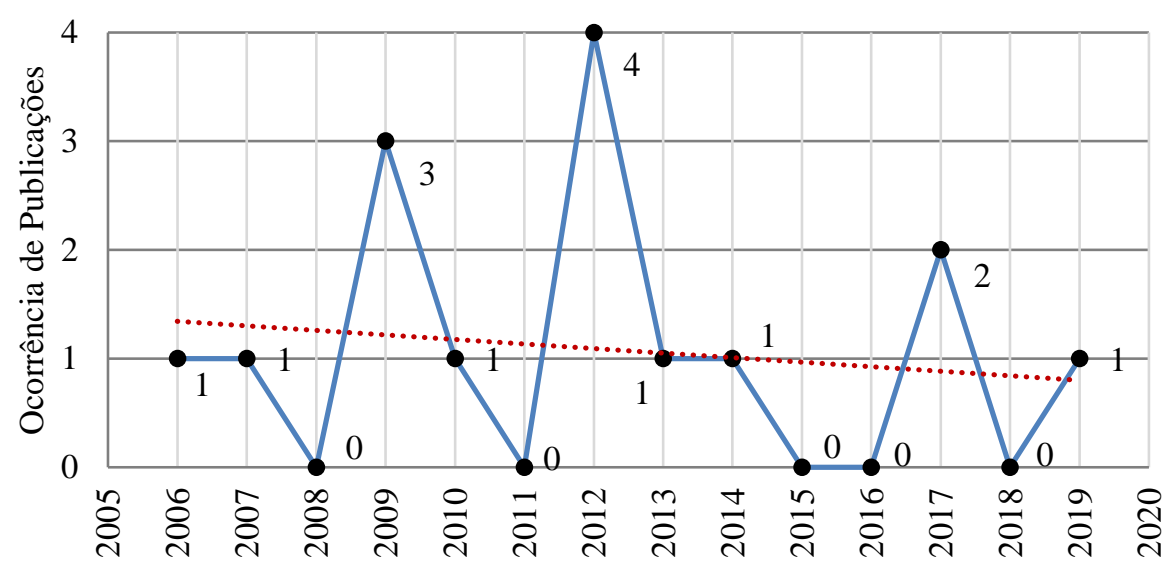

A Tabela 3 apresenta o número de ocorrência de publicações por autor e países. Destacase que John Gambatese lidera nas pesquisas com oito artigos publicados acerca da temática nos Estados Unidos. Verificou-se também a existência de coautorias entre os profissionais resultando em melhoras nas publicações.

Tabela 3 - número de artigos por autor

\begin{tabular}{llc}
\hline \multicolumn{1}{c}{ Autor } & \multicolumn{1}{c}{ País } & Artigos \\
\hline GAMBATESE, J. A. & Estados Unidos & 8 \\
BEHM, M. & Estados Unidos & 5 \\
RAJENDRAN, S. & Estados Unidos & 4 \\
HALLOWELL, M. & Estados Unidos & 4 \\
KARAKHAN, A. A. & Estados Unidos & 2 \\
FORTUNATO, B. R. & Estados Unidos & 2 \\
DEWLANEY, K. S. & Estados Unidos & 2 \\
CHEN, H. & Estados Unidos & 1 \\
TYMVIOS, N. & Estados Unidos & 1 \\
ALBATTAH, M. & Estados Unidos & 1 \\
LENTZ, T. & Estados Unidos & 1 \\
HEIDEL, D. & Estados Unidos & 1 \\
ROUCHERAY, M. & Estados Unidos & 1 \\
ONUBI, H. O. & Malásia & 1 \\
YUSOF, N. A. & Malásia & 1 \\
SANUSI HASSAN, A. & Malásia & 1 \\
RABBANI, E. R. K. & Brasil & 1 \\
LIMA, A. N. F. & Brasil & 1 \\
SANTOS, F. J. B. & Brasil & 1 \\
COSTA, V. A. S. & Brasil & 1 \\
MALAFAYA-BAPTISTA, F. & Brasil & 1 \\
\hline
\end{tabular}

\subsection{Discussões dos artigos encontrados}

Edifícios verdes não são construídos com medidas adicionais de segurança e saúde no projeto e no processo de planejamento. As empresas de construção selecionadas para construir edifícios verdes não são obrigadas a terem sistemas especiais de gerenciamento de segurança ou evidências de um determinado desempenho de segurança (BEHM et al., 2009). 
Conforme Rajendran e Gambatese (2009a), questionam que no caso de ocorrer lesão ou fatalidade durante a construção de um edifício projetado para ser sustentável, esse projeto deveria ser considerado sustentável? Rajendran e Gambatese (2009a), acreditam que a SST são partes do processo de sustentabilidade de uma edificação. Argumentam que os projetos não podem ser intitulados como sustentáveis caso a SST não forem levadas em consideração durante todo o ciclo de vida da edificação desde sua construção até a demolição.

Rajendran e Gambatese (2009a) construíram uma proposta de 50 itens que que denominaram - "Sustainable Construction Safety and Health (SCSH)" derivados de uma pesquisa de opinião com especialistas em construção nos Estados Unidos. A proposta da pesquisa é disponibilizar os itens de segurança e saúde no sistema para implementação em todo o ciclo da edificação, podendo inclusive, sofrer permanentes alterações ao longo das aplicações.

Rajendran et al. (2009b) investigaram os impactos dos projetos e construções sustentáveis na SST. Uma análise estatística abrangendo 86 projetos, sendo 38 considerados verdes e 48 não verdes, ou convencionais. Concluíram que havia pouca ou nenhuma diferença entre as obras sustentáveis (verdes) e obras convencionais no aspecto da SST e não foi possível relacionar os acidentes de trabalho especificamente às obras sustentáveis. O esperado era que os acidentes nas construções verdes fossem menores, e por este motivo, é preciso inserir elementos de avaliação de SST nas certificações.

Conforme Gambatese et al. (2006) existem evidências de impactos positivos e negativos na SST nas construções de edifícios sustentáveis. Os impactos negativos devem ser mitigados através da integração do conceito de segurança e saúde com as práticas da construção verde. Entretanto sugere que mais pesquisas são necessárias para documentar todos os impactos positivos e negativos.

Fortunato III et al. (2012) ao analisar o resultado da pesquisa de Rajendran et al. (2009b), apresentaram seis estudos de caso detalhados e dois estudos de caso de validação, conduzidos um protocolo pré-estabelecido, para avaliar o real motivo das obras sustentáveis (verdes) e obras convencionais, no aspecto da SST, apresentarem quantidades semelhantes acidentes, e em alguns casos, até mesmo as obras certificadas (verdes) apresentarem número de acidentes maiores. Os resultados indicam que, para os casos analisados, (1) os trabalhadores em projetos de obras sustentáveis são mais expostos a trabalhos em altura, principalmente com eletricidade, e quando no solo, estão perto de equipamentos pesados por um período maior do que os trabalhadores em obras convencionais; (2) observaram também, que os trabalhadores são expostos a mais tarefas de alto risco, como construção de átrios, instalação de telhados verdes e instalação de painéis fotovoltaicos.

Dewlaney e Hallowell (2012a), entrevistaram 11 construtores, 13 projetistas e 02 subconstrutores e revelaram que 14 créditos da certificação LEED possui pelo menos um método de construção ou estratégia para projeto de prevenção que pode ser usado para mitigar os riscos identificados. Essa tentativa de identificar os riscos durante a fase de projeto, provavelmente terá um impacto positivo na segurança e saúde dos trabalhadores durante a construção. Também constataram que existem diversos métodos para alcançar os créditos LEED preservando a segurança e a saúde apenas selecionando estrategicamente os métodos usados para ganhar cada crédito. Dentre as recomendações Dewlaney e Hallowell (2012a), se destacam: (1) concepção de projetos com layouts espaciais que removem as condições perigosas; (2) utilizar materiais pré-fabricados; (3) atenção especial para o trabalho em ambientes externos, com riscos associados à construção em altura, sobrecarga, (4) atenção especial para com sistemas elétricos energizados e; (4) trabalhos em espaços confinados.

Dewlaney et al. (2012b) observaram que alguns créditos aumentam os riscos de SST. Todavia, é possível obter o nível de certificação mais alto (LEED Platinum) sem implementar apenas cinco créditos dos 14 analisados. Dessa forma, seria reduzida drasticamente a possibilidade de ocorrer algum tipo de acidente. 
Gambatese et al. (2007), com o objetivo de quantificar os riscos adicionais devido as técnicas das obras sustentáveis, realizaram 26 entrevistas com projetistas e construtores que completaram em média quatro projetos de edifícios verdes. Os resultados indicam que os elementos de projeto e os métodos de construção implementados para atingir 12 dos 49 créditos LEED aumentam a frequência de lesões ou exposição a ambientes conhecidos de alto risco. Os impactos mais significativos foram um aumento de $36 \%$ nas lacerações, tensões e entorses decorrentes da reciclagem de materiais de construção; um aumento de $24 \%$ nas quedas principalmente devido à instalação de energia solar fotovoltaica; um aumento de $19 \%$ na fadiga ocular ao instalar membranas reflexivas de teto; e um aumento de $14 \%$ na exposição a substâncias nocivas ao instalar tecnologias inovadoras de águas residuais. Gambatese et al. (2007) concluem que 14 créditos são os principais responsáveis pelos acidentes, e que é possível chegar à certificação sem utilizar dos referidos créditos.

Karakhan e Gambatese (2017a), investigaram a percepção de dois grupos (projetista e construtores) sobre projeto de prevenção de SST como requisitos do sistema de classificação do LEED. No grupo dos construtores $69 \%$ afirmaram que considerariam buscar os créditos de SST como parte da certificação LEED em suas futuras construções, e apenas $6 \%$ mostraram resistência a ideia. Já no grupo dos projetistas apenas $47 \%$ indicaram que considerariam a implementação dos requisitos de crédito SST em seus futuros projetos sustentáveis. Para alguns dos itens de SST o grupo dos construtores foi até 3,59 vezes mais propensos em aceitar a implementação dos créditos pilotos que os projetistas (Arquitetos e Engenheiros). No artigo, os créditos pilotos são tratados como PtD (LEED Prevention Through Design).

Karakhan e Gambatese (2017b) informam que a saúde e segurança do trabalhador é uma dimensão integral da sustentabilidade social. Assim, pesquisaram metodologias para avaliar, quantificar e classificar o risco de saúde e segurança ocupacional associado à construção, operação e manutenção (todo o ciclo de vida) de projetos sustentáveis na indústria de construção civil dos Estados Unidos e realizaram uma comparação com os riscos encontrado em projetos convencionais. Os estudos revelaram que alguns elementos de projetos e de práticas de construções utilizadas na certificação LEED podem impactar negativamente na SST. Dos 54 créditos disponíveis nas categorias principais LEED (2009) todos foram analisados por Karakhan e Gambatese (2017b), a maioria dos créditos (37 créditos) não representou impacto positivo ou negativo na SST dos trabalhadores da construção e posteriormente da manutenção quando comparados com os projetos tradicionais. No entanto, 12 créditos foram considerados como tendo um impacto potencial na saúde e segurança do trabalhador. Destacando-se o Efeito Ilha de Calor e créditos de Gerenciamento de Resíduos de Construção, que foram considerados geradores de risco de segurança inaceitável para o pessoal de construção e/ou manutenção. Sugerem assim, atenção especial e imediata por parte tanto dos projetistas como dos construtores. Apenas quatro créditos foram encontrados para abordar explicitamente a SST, mas dois deles não são frequentemente obtidos por projetos sustentáveis, segundo a análise de Karakhan e Gambatese (2017b).

Albattah et al. (2013), desenvolveram um programa piloto que fornece orientações específicas de prevenção através do projeto, e também gerencia a segurança de construção para cada crédito do LEED. Nesse ensaio participaram 16 profissionais com mais de 18 anos de experiência e foi verificado que alguns créditos apresentaram maior risco à SST, porém o aumento da taxa de lesões não foi associado ao LEED.

Gambatese e Tymvios (2012) revisaram os créditos da Certificação LEED-NC v2 e constataram que podem afetar positivamente, a maioria dos créditos, e negativamente a SST. Os riscos que impactam diretamente a SST depende do tipo do elemento que está sendo construído e de como ele é executado. Dependendo do serviço a ser realizado, não apresentaram diferenças das construções convencionais. Do total de 35 créditos, apenas seis aumenta o risco de acidente. 
Segundo Helen Chen (2010), da forma como são construídos e projetados os edifícios sustentáveis, podem causar ferimentos e doenças aos trabalhadores, tanto quanto os edifícios tradicionais.

Onubi et al. (2019) analisaram três efeitos da adoção de práticas verdes (gerenciamento de águas pluviais, gestão de resíduos e de energia) de construção no desempenho de saúde e segurança dos projetos de construção. Concluíram que, entre as várias práticas de canteiro de obras verdes, o desempenho de saúde e a segurança não se aplicam a todos os créditos e/ou prérequisitos da certificação, os quais podem depender das peculiaridades do projeto e dos conjuntos de habilidades dos trabalhadores.

Rabbani et al. (2014) aplicaram um formulário de pesquisa sobre a segurança dos trabalhadores envolvidos em obras em processo de certificação no Brasil em 2013. Foram entrevistados os engenheiros residentes das obras, os supervisores de segurança do trabalho e responsáveis pela implantação do sistema de acreditação. Rabbani et al. (2014) também realizaram visitas às obras e análise documental, e como conclusão da pesquisa, observaram que muitas das medidas aplicadas nos processos de construção contribuíram positivamente para a melhoria da qualidade do meio ambiente e para a segurança do trabalhador devido à melhor organização e resultando em fiscalização constante, derivada do processo LEED.

\section{CONCLUSÃO}

As certificações estão exigindo cada vez mais dos empreendedores nas questões ambientais e também, no bem-estar dos ocupantes dos empreendimentos. Entretanto, de acordo com os trabalhos analisados, em alguns casos, obras certificadas apresentaram maiores índices de acidentes do trabalho que as obras convencionais. Isso ocorreu pelo principalmente pelo aumento do trabalhado em altura, pela inserção de telhados verdes ou painéis fotovoltaicos, bem como pelo aumento do trabalho com vidros.

Os artigos encontrados trataram principalmente de analisar as certificações LEED, pois os grupos de pesquisa localizados que trabalham com o nexo entre a SST e as obras certificadas estão predominantemente nos EUA. Sendo, portanto, umas das recomendações é realizar a pesquisa sobre a relação entre a Saúde e Segurança do Trabalho (SST) e outras certificações.

Embora as certificações em construções sustentáveis estejam em constante evolução, uma hipótese considera para a pesquisa, era de que as obras de edificações certificadas fossem mais seguras para os trabalhadores que as convencionais. Entretanto, as pesquisas do grupo do pesquisador liderado por John A. Gambatese demonstraram que, em diversos casos, ocorreu o contrário do esperado, as obras de edificações certificadas tiveram número de acidentes do trabalho maior que as convencionais. Por este motivo, propostas de créditos pilotos para a certificação LEED foram apresentadas visando a implementação das preocupações com a SST.

Os grupos de pesquisas que publicaram a maior parte dos trabalhos localizados nesta revisão são dos Estados Unidos. Assim, os artigos revisados em sua grande maioria demostram a realidade da sua localidade.

No caso brasileiro, somente foi localizado um trabalho que faz a discussão do nexo entre a SST e as obras certificadas. O trabalho não é conclusivo neste item, mas Rabbani et al. (2014) apontaram que as certificações por apenas exigirem e fiscalizarem os responsáveis pelo empreendimento para o cumprimento das legislações e normas municipais, estaduais e federais, já contribuem com o aumento da segurança nas obras certificadas, para o caso brasileiro.

Observa-se que a SST deve ser parte do sistema de certificação de construção sustentável, bem como, os projetos de prevenção contribuem de forma positiva. Assim, até o presente momento, não é possível determinar se as construções sustentáveis são mais seguras que as convencionais em nível mundial. Entretanto, no caso brasileiro, as referências analisadas observaram que muitas das medidas aplicadas nos processos de construção contribuíram 
Silva, H. C. C.; Souza, D. F.; Guarda, E. L. A.; As obras de edifícios sustentáveis são mais seguras que as construções convencionais? Uma revisão. ES - Engineering and Science, 2021, 10:3.

positivamente para a melhoria da qualidade do meio ambiente e para a segurança do trabalhador devido à melhor organização e resultando em fiscalização constante.

Diante disso, recomenda-se que pesquisas nessas abordagens seriam necessárias, considerando as atuais certificações ambientais, e a ciência de que para que uma edificação seja considerada sustentável é necessária existam profundas preocupações com a segurança e saúde dos trabalhadores e usuários ao longo de todo o ciclo de vida da edificação, para que o processo verde seja verdadeiramente classificado como sustentável.

\section{REFERÊNCIAS}

AEAT. ANUÁRIO ESTATÍSTICO DE ACIDENTES DO TRABALHO. Anuário Estatístico de Acidentes do Trabalho: AEAT 2018. Ministério da Fazenda. Brasília: MF, 2018. 1288 p.

ALBATTAH, M.; ROUCHERAY, M; HALLOWELL, M. Sustainable buildings: applying prevention through design. Professional Safety, 2013. June:76-80.

BEHM, M.; LENTZ, T.J.; HEIDEL, D.; GAMBATESE, J. Prevention through design and green buildings: A U.S. perspective on collaboration. Proceedings of working together: Planning, designing and building a health and safe construction industry, In: International Council for Research and Innovation in Building and Construction (CIB) W99 Conference. Melbourne, Australia, 2009.

BRE. BUILDING RESEARCH ESTABLISHMENT. BREEAM UK New Construction 2018. Technical Manual. 2018. Disponível em: https:// https://www.breeam.com/NC2018/. Acesso em: 16 dez. 2019.

CAMPOS, P. A. M. Cimento de baixo impacto ambiental produzido com resíduos cindustriais e de mineração. Monografia, f.64 (Graduação) - Curso de Engenharia Civil, Universidade Federal de Ouro Preto. Ouro Preto, 2012.

DEWLANEY, K.; HALLOWELL, M. Prevention through design and construction safety management strategies for high performance sustainable building construction. Construction Management and Economics, 2012a. 30. 165-177. DOI: 10.1080/01446193.2011.654232.

DEWLANEY, K.; HALLOWELL, M.; FORTUNATO III, B.R. Safety Risk Quantification for High Performance Sustainable Building Construction. Journal of Construction Engineering and Management, 2012b. 138. 964-971. 10.1061/(ASCE)CO.1943-7862.0000504.

DGNB. GERMAN SUSTAINABLE BUILDING COUNCIL. Criteria. Disponível em: https://www.dgnb-system.de/en/buildings/new-construction/criteria/. [2018?]. Acesso em: 16 dez. 2019.

ECA. EUROPEAN CONCERTED ACTION. Cost Project 613. Report No 4. Sick Building Syndrome. Commission of the European Communities. Luxembourg. 1989.

EPA. UNITED STATES ENVIRONMENTAL PROTECTION AGENCY. Buildings and Infrastructure from a Sustainability Perspective. 2014. Disponível em: https://www.epa.gov/sites/production/files/2016-

09/documents/buildingsandinfrastructurefromasustainabilityperspective.pdf. Acesso em: 12 nov. 2019.

- Definition of Green Building. Disponível em:

https://archive.epa.gov/greenbuilding/web/html/about.html. [2014]. Acesso em: 13 dez. 2019.

FARIA, F. C; SCHMID, A. L. Avaliação do Comportamento de tintas naturais para construção civil frente ao intemperismo através de ensaio de envelhecimento acelerado. In: Encontro 
Silva, H. C. C.; Souza, D. F.; Guarda, E. L. A.; As obras de edifícios sustentáveis são mais seguras que as construções convencionais? Uma revisão. ES - Engineering and Science, 2021, 10:3.

\section{Nacional de Conforto no Ambiente Construído e Encontro Latino Americano de Conforto no Ambiente Construído. Anais...2015.}

FORTUNATO III, B.R.; HALLOWELL, M.R.; BEHM, M.; DEWLANEY, K. Identification of Safety Risks for High-Performance Sustainable Construction Projects. Journal of Construction Engineering and Management, 2012. 138. 499-508. 10.1061/(ASCE) CO.1943-7862.0000446.

FUNDAÇÃO VANZOLINI. O processo de certificação. Processo AQUA. Disponível em: https://vanzolini.org.br/aqua/certifique-o-seu-empreendimento/. [2016]. Acesso em: 16 dez. 2020.

GAMBATESE, J.A.; RAJENDRAN, S.; BEHM, M.G. Building toward sustainable safety and health. In: ASSE Professional Development Conference. American Society of Safety Engineers: Seattle, 2006.

GAMBATESE, J.A.; RAJENDRAN, S.; BEHM, M.G. Green Design \& Construction: Understanding the effect on construction worker safety and health. Professional Safety. May Issue, 28-35, ASSE, 2007.

GAMBATESE, J.; TYMVIOS, T. LEED Credits: How They Affect Construction Worker Safety. Professional Safety, October Issue, 42-52, ASSE, 2012.

GBC. GREEN BUILDING COUNCIL BRASIL. Guia rápido. Certificação GBC Brasil Casa. 2017. Disponível em: https://www.gbcbrasil.org.br/certificacoes/. Acesso em: 15 dez. 2020.

Brasil ocupa o $4^{\circ}$ lugar no ranking mundial de construções sustentáveis certificadas pela ferramenta internacional LEED. 2018. Disponível em: https://www.gbcbrasil.org.br/brasil-ocupa-o-4olugar-no-ranking-mundial-de-construcoes-sustentaveis-certificadas-pela-ferramenta-internacionalleed/. Acesso em: 04 fev. 2021.

CHEN, H. Green and Healthy Jobs. Labor Occupational Health Program. The Center for Construction Research and Training Report. University of California: Berkeley, 2010.

INGRAO, C; SCRUCCA, F; TRICASE, C; ASDRUBALI, F. A comparative Life Cycle Assessment of external wall-compositions for cleaner construction solutions in buildings. Journal Of Cleaner Production, v. 124, p. 283-298. Elsevier BV, 2016.

KARAKHAN, A.A.; GAMBATESE, J.A. Integrating worker health and safety into sustainable design and construction: designer and constructor perspectives. Journal of Construction Engineering and Management. 143 (9), 04017069, 2017a.

KARAKHAN, A.A.; GAMBATESE, J.A. Identification, quantification, and classification of potential safety risk for sustainable construction in the United States. Journal of Construction Engineering and Management. 143 (7), 04017018, $2017 \mathrm{~b}$.

MARTÍN-MARTÍN, Alberto et al. Google Scholar, Web of Science, and Scopus: A systematic comparison of citations in 252 subject categories. Journal of informetrics, v. 12, n. 4, p. 1160-1177, 2018.

MOHER, D.; LIBERATI, A.; TETZLAFF, J.; ALTMAN, D.G. Preferred Reporting Items for Systematic Reviews and Meta-Analyses: The PRISMA Statement. 2009. PLoS Med 6 (7): e1000097. https://doi.org/10.1371/journal.pmed.1000097.

NIOSH. THE NATIONAL INSTITUTE FOR OCCUPATIONAL SAFETY AND HEALTH. NIOSH perspectives on sustainable buildings: green and safe. 2010. Disponível em: https://www.cdc.gov/niosh/topics/greenconstruction/pdfs/NIOSHperspectiveOnSustainableBuildings. pdf. Acesso em: 17 dez. 2020. 
ONUBI, H.O.; YUSOF, N.; HASSAN, A.S. Adopting green construction practices: health and safety implications. Journal of Engineering, Design and Technology, Vol. 18 No. 3, p. 635-652, 2019.

RABBANI, E.R.K.; LIMA, A.N.F.; SANTOS, F.J.B.; COSTA, V.A.S.; MALAFAYA-BAPTISTA, F. Sustainability Measures and Potential Impacts on Occupational Safety and Health: Brazilian Constructions. 2014. EJGE. 19. 17675.

RAJENDRAN, S.; GAMBATESE, J. Development and Initial Validation of Sustainable Construction Safety and Health Rating System. Journal of Construction Engineering and Management. 135. 10.1061/(ASCE)0733-9364(2009)135:10(1067), 2009a.

RAJENDRAN, S.; GAMBATESE, J.A.; BEHM, M.G. Impact of green building and construction on worker safety and health. Journal of Construction Engineering and Management. DOI: 10.1061/(ASCE)0733-9364(2009)135:10(1058), 2009b.

USGBC. UNITED STATE GREEN BUILDING COUNCIL. LEED v4.1 Building Design and Construction. [2020]. Disponível em: https://build.usgbc.org/bdc41. 2021. Acesso em: 02 fev. 2021.

Prevention through Design. 2015. Disponível em: http://www.usgbc.org/credits/preventionthroughdesign. Acesso em: 17 dez. 2020.

. LEED by the numbers: 16 years of steady growth. 2016. Disponível em: https://www.usgbc.org/articles/leed-numbers-16-years-steady-growth. Acesso em: 31 jan. 2021. 